\title{
Modulation of EZH2 expression in T cells improves efficacy of anti-CTLA-4 therapy
}

\author{
Sangeeta Goswami, ${ }^{1}$ Irina Apostolou, ${ }^{2}$ Jan Zhang, ${ }^{1}$ Jill Skepner, ${ }^{2}$ Swetha Anandhan, ${ }^{1}$ Xuejun Zhang, ${ }^{3}$ Liangwen Xiong, ${ }^{1}$ \\ Patrick Trojer, ${ }^{2}$ Ana Aparicio, ${ }^{1}$ Sumit K. Subudhi, ${ }^{1}$ James P. Allison, ${ }^{3,4}$ Hao Zhao, ${ }^{3}$ and Padmanee Sharma ${ }^{1,3,4}$
}

'Department of Cenitourinary Medical Oncology, The University of Texas MD Anderson Cancer Center, Houston, Texas, USA. ${ }^{2}$ Constellation Pharmaceuticals, Cambridge, Massachusetts, USA. ${ }^{3}$ mmmunotherapy Platform, The University of Texas MD Anderson Cancer Center, Houston, Texas, USA. ${ }^{4}$ Department of Immunology, The University of Texas MD Anderson Cancer Center, Houston, Texas, USA.

\begin{abstract}
Enhancer of zeste homolog 2-mediated (EZH2-mediated) epigenetic regulation of T cell differentiation and Treg function has been described previously; however, the role of EZH2 in T cell-mediated antitumor immunity, especially in the context of

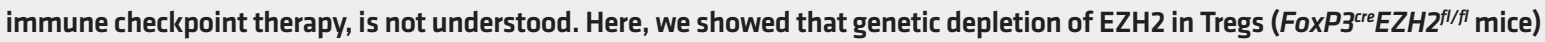
leads to robust antitumor immunity. In addition, pharmacological inhibition of EZH2 in human T cells using CPI-1205 elicited phenotypic and functional alterations of the Tregs and enhanced cytotoxic activity of Teffs. We observed that ipilimumab (anti-CTLA-4) increased EZH2 expression in peripheral T cells from treated patients. We hypothesized that inhibition of EZH2 expression in T cells would increase the effectiveness of anti-CTLA-4 therapy, which we tested in murine models. Collectively, our data demonstrated that modulating EZH2 expression in T cells can improve antitumor responses elicited by anti-CTLA-4 therapy, which provides a strong rationale for a combination trial of CPI-1205 plus ipilimumab.
\end{abstract}

\section{Introduction}

Enhancer of zeste homolog 2 (EZH2) is expressed in T cells following its activation via CD28 signaling (1). EZH2 is a catalytic subunit of polycomb repressive complex 2 (PRC2) that trimethylates lysine 27 on histone H3 (H3K27me3), leading to gene repression $(2,3) . \mathrm{EZH} 2$ can form a complex with FoxP3 that is necessary for maintaining the identity of naturally occurring Tregs following its activation $(4,5)$. Further, EZH2 can inhibit $\mathrm{T}$ cell differentiation into Teffs, mostly by conferring repressive marks in key cytokine and transcription factor genes (5-10). Overall, EZH2 maintains suppressive activity in T cells that prevents the development of autoimmunity $(11,12)$, but may have a negative impact on antitumor immunity. EZH2 has been shown to regulate chemokine expression in cancer cells and immunogenicity of melanoma tumor cells; thereby, EZH2 inhibition can increase $\mathrm{T}$ cell infiltration (13-15). However, the direct role of EZH2-mediated reprogramming of T cells in antitumor immunity, especially in the context of immune checkpoint therapy, has not been studied.

Here, we show that genetic depletion of EZH2 in Tregs (using FoxP $3^{\text {cre }} \mathrm{EZH} 2^{f / f l}$ mice) and pharmacological inhibition of EZH2 by CPI-1205 elicits phenotypic and functional alterations of Tregs, leading to an effector-like T cell profile. Further, CPI-1205 enhances the cytotoxicity of human Teffs in vitro and the propor-

Conflict of interest: PS has consulted for Constellation Pharmaceuticals, Jounce Therapeutics, Kite Pharma, Evelo, Astellas Pharma, AstraZeneca, Amgen, ClaxoSmithKline, and Bristol-Myers Squibb. PS serves on the scientific advisory boards for Jounce Therapeutics, Neon, Constellation Pharmaceuticals, and Kite Pharma. PS is a cofounder of Jounce Therapeutics. PT, JS, and IA serve in the translational research division of Constellation Pharmaceuticals.

Submitted: January 11, 2018; Accepted: June 12, 2018.

Reference information: J Clin Invest. 2018;128(9):3813-3818.

https://doi.org/10.1172/JCI99760. tion of tumor-infiltrating cytotoxic T cells in vivo in the murine model. We observed that ipilimumab (a fully human monoclonal immunoglobulin G1 antibody that blocks cytotoxic T lymphocyte associated protein 4 [CTLA-4]) increases EZH2 expression in human T cells across various tumor types and that increased EZH2 expression in $\mathrm{T}$ cells inversely correlates with clinical outcome in a cohort of patients with prostate cancer. On this basis, we postulated that upregulation of EZH2 mediated by anti-CTLA-4 in T cells modulates $\mathrm{T}$ cell responses and diminishes the effectiveness of anti-CTLA-4 therapy. Consistent with our hypothesis, we found that pharmacologic inhibition of EZH2 by CPI-1205 increases effector-like $\mathrm{T}$ cell responses and enhances effectiveness of antiCTLA-4 therapy in tumor-bearing mice.

\section{Results and Discussion}

To interrogate the role of EZH2 in T cell-mediated antitumor immunity, we used tumor-bearing FoxP $3^{\text {cre }} E Z H 2^{f / f l} \mathrm{C} 57 \mathrm{BL} / 6$ mice, which lack EZH2 specifically in the Tregs. FoxP $3^{\text {cre }} \mathrm{EZH} 2^{f / / f l}$ mice and WT control C57BL/6 mice were inoculated with a murine bladder cancer cell line (MB49). Tumor growth and immune profiles were studied. FoxP $3^{\text {cre }} \mathrm{EZH} 2^{f / f l}$ mice had significantly less tumor growth compared with the control mice (Figure 1A). Analysis of the tumor microenvironment showed a proinflammatory state evidenced by increased $\mathrm{CD} 8^{+} \mathrm{IFN}-\gamma^{+}$cells, $\mathrm{CD} 8^{+}$granzyme B-positive $\left(\mathrm{GzB}^{+}\right)$cells, and $\mathrm{CD}^{+} \mathrm{TNF}^{-} \alpha^{+}$cells in tumor-bearing FoxP $3^{\text {cre }} \mathrm{EZH} 2^{f / f l}$ mice compared with the control mice (Figure 1B). Increased frequency of cytokine-producing $\mathrm{CD} 8^{+} \mathrm{T}$ cells in the FoxP $3^{\text {cre }} E Z H 2^{f l / f l}$ mice suggested EZH2 inhibition in Tregs also have an indirect positive impact on $\mathrm{CD}^{+} \mathrm{T}$ cells. Importantly, we observed increased intratumoral abundance of FoxP3 $3^{+}$IFN- $\gamma^{+}$ double-positive cells, which implied that the loss of EZH2 function in Tregs can reprogram their phenotype to effector-like T cells and induce robust antitumor immunity (Figure $1 \mathrm{C}$ and Supplemental 
A

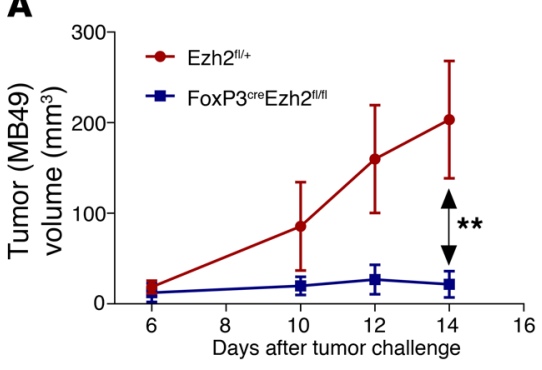

B

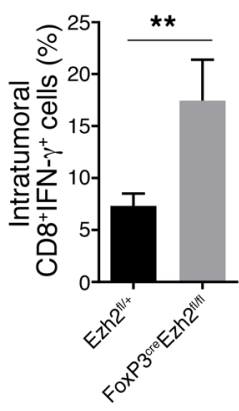

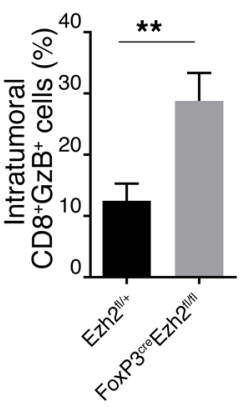

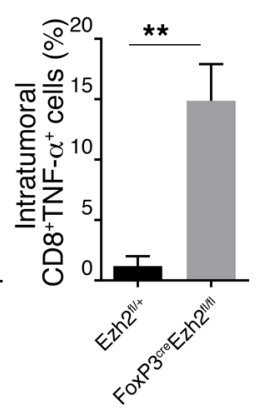

C

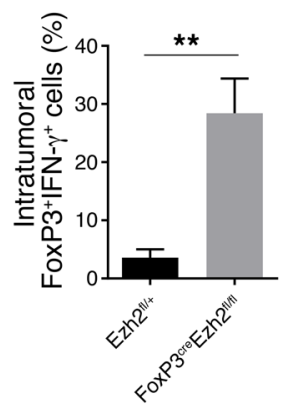

D

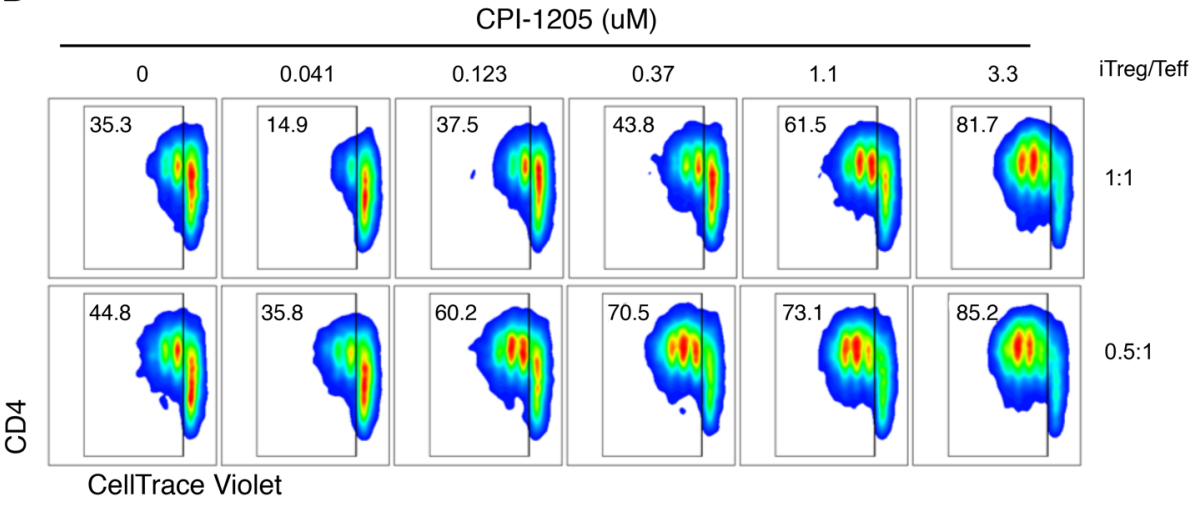

E

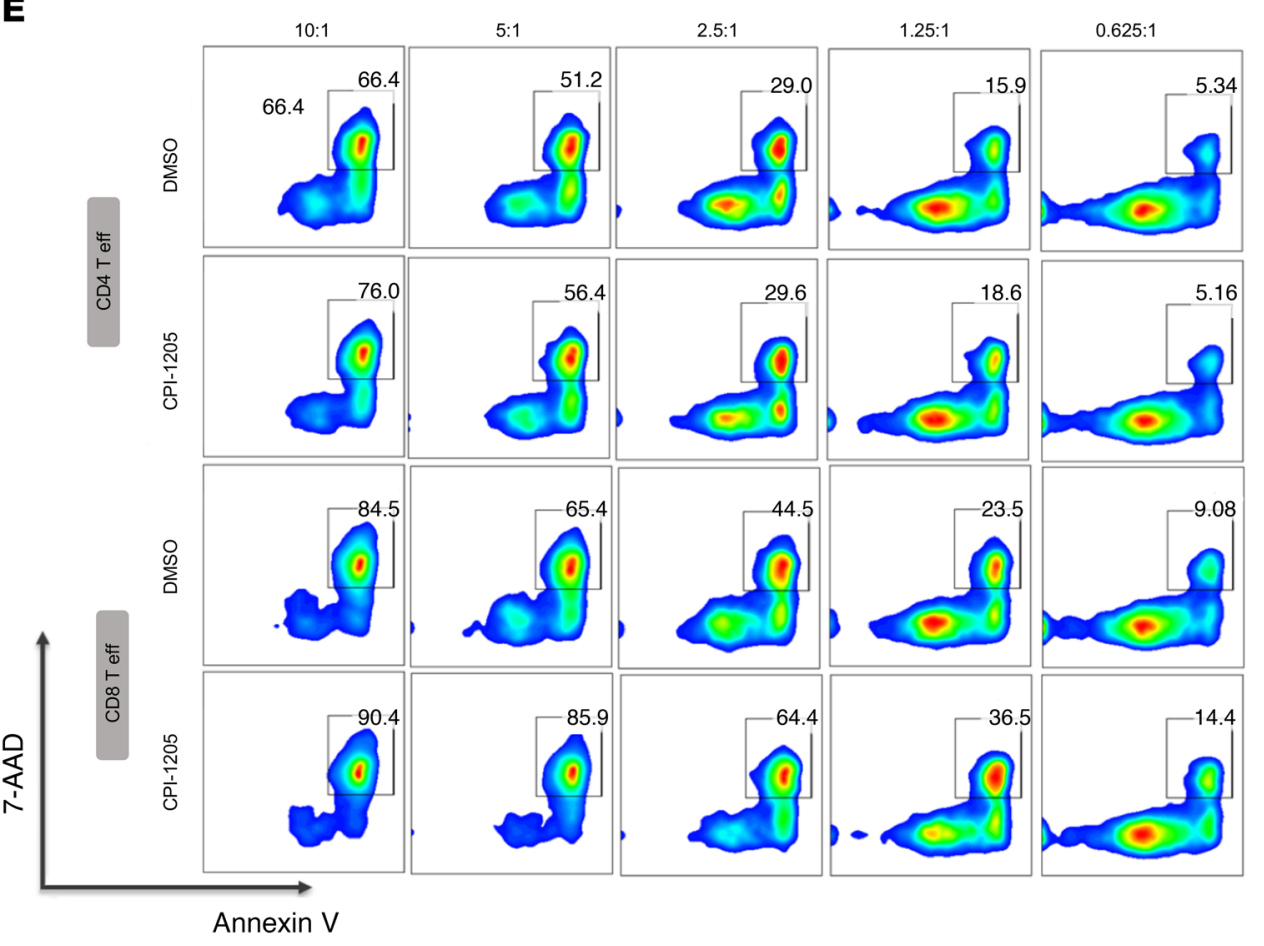

Figure 1. EZH2 inhibition by CPI-1205 enhances $\mathrm{T}$ cell-mediated antitumor immunity. Tumor growth (A) and percentages of intratumoral CD8 $8^{+} \mathrm{FN}-\gamma^{+}$ cells, $\mathrm{CD}^{+} \mathrm{CzB}^{+}$cells, and CD8 ${ }^{+} \mathrm{TNF}-\alpha^{+}$ (B) and FoxP3 ${ }^{+} \mathrm{IFN}-\gamma^{+}$cells (C) in MB49 tumor-bearing $\mathrm{EZH}^{f /++}(n=10)$ and FoxP3 ${ }^{C r e} E Z H 2^{f / / f l}(n=10)$ mice. (D) Differentiated human iTregs with or without CPI-1205 were cultured with CellTrace Violet-labeled Teffs for 72 hours at various ratios. Data are presented as CellTrace Violet ${ }^{+}$proliferating Teffs at different concentrations of CPI-1205. (E) Preactivated human $\mathrm{CD}^{+}$and CD8 ${ }^{+} \mathrm{T}$ cells (with or without CPI-1205) were cultured with CellTrace Violetlabeled Nalm-6 target cells along with blinatumomab. Data are presented as percentages of Annexin $7 A A D^{+}$cells at various ratios of effector/target cells. Data are representative of 3 independent experiments. A 2-tailed Student's $t$ test was used to determine significance; ${ }^{* *} P<0.01$.

Figure 1; supplemental material available online with this article; https://doi.org/10.1172/JCI99760DS1).

To investigate whether the data observed in the FoxP3 ${ }^{\text {cre }} \mathrm{EZH} 2^{\text {ff/fl }}$ mouse model could be recapitulated by pharmacological inhibition of EZH2 in T cells, we compared the effect of different EZH2 inhibitors on Treg differentiation. We compared a novel agent, CPI-1205, with commercially available EZH2 inhibitors. We found that CPI-
1205 had the most striking effect on suppression of murine inducible regulatory $\mathrm{T}$ cell (iTreg) differentiation compared with the other EZH2 inhibitors, and none of the inhibitors had a significant effect on Treg proliferation (Supplemental Figure 2, A and B). Next, we interrogated the effect of CPI-1205 on human Treg differentiation and function. CPI-1205 had a milder effect on iTreg differentiation in the human system, albeit a dose-dependent decrease in FoxP3 
A

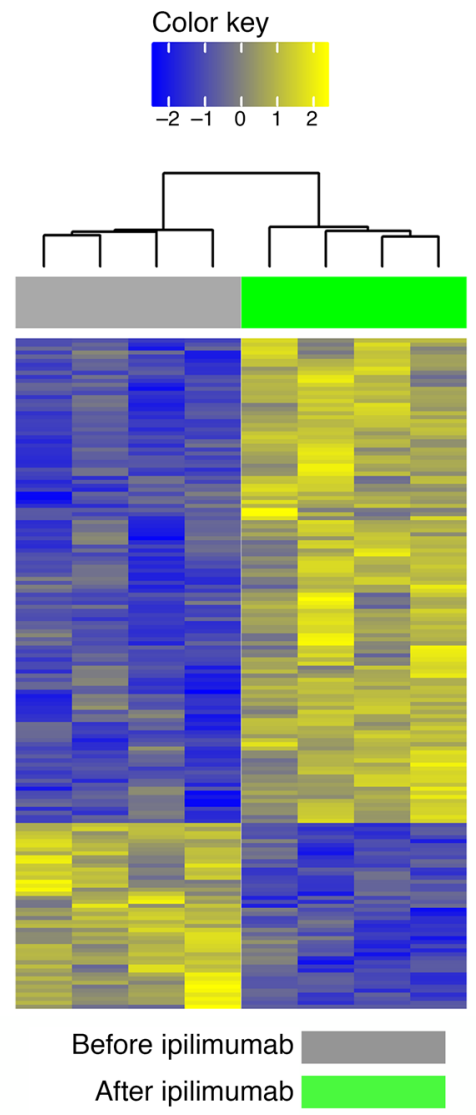

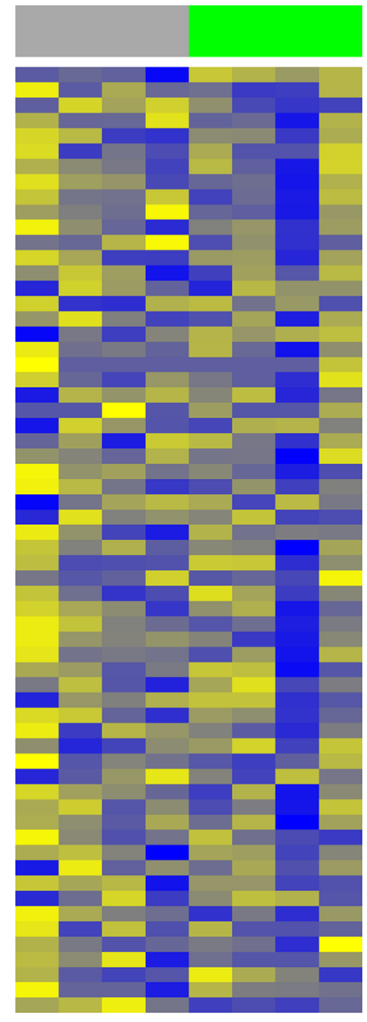

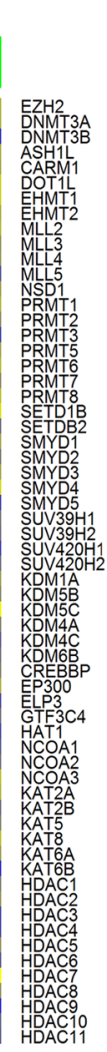

B

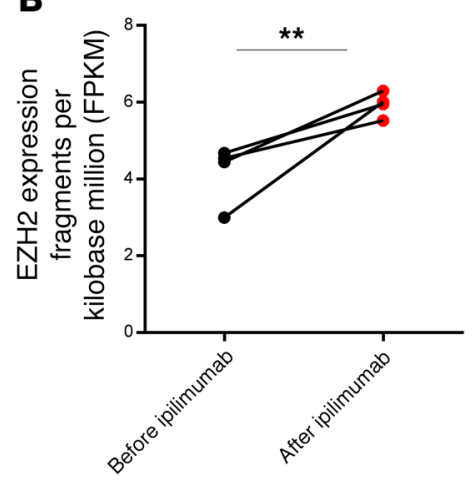

C

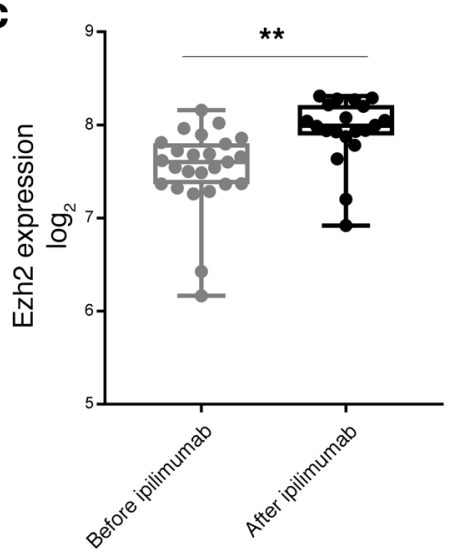

Figure 2. Ipilimumab increases EZH2 expression in T cells. (A) Unsupervised hierarchical cluster analysis of RNA sequencing data from the peripheral $\mathrm{CD}^{+} \mathrm{T}$ cells of patients with metastatic melanoma before and after 3 doses of ipilimumab therapy (week 7). (B) Matched, paired analysis of EZH2 expression before and after ipilimumab therapy. (C) EZH2 expression in peripheral CD4+ $\mathrm{T}$ cells from patients with metastatic prostate cancer at baseline and after 3 doses of ipilimumab therapy. A 2-tailed Student's $t$ test was used to determine significance; ${ }^{* *} P<0.01$.

expression was observed (Supplemental Figure 3, A and B). We performed suppression assays using human iTregs differentiated in the presence or absence of CPI-1205. Human peripheral blood mononuclear cell-derived naive $\mathrm{T}$ cells were differentiated into iTregs in the presence of DMSO or CPI-1205. Differentiated Tregs were then cultured with CellTrace Violet-labeled conventional T cells (Teffs) for 72 hours at various ratios of iTregs/Teffs. We found that iTregs lose their suppressive capacity after treatment with CPI1205 as evidenced by increased proliferation of Teffs (Figure 1D). Loss of suppressive activity was noted even at concentrations that didn't impact iTreg differentiation.

To understand CPI-1205-mediated molecular changes underlying the loss of suppressive activity of Tregs, we used naive murine $\mathrm{T}$ cells $\left(\mathrm{CD} 4^{+} \mathrm{CD} 25^{\text {low }} \mathrm{CD} 44^{\text {low }} \mathrm{CD} 62^{\text {hi }}\right)$ from FoxP3enhanced green fluorescent protein (eGFP) C57BL/6 mice that were differentiated into iTregs in the presence or absence of CPI1205. Purified $\mathrm{GFP}^{+} \mathrm{FoxP}^{+}$Tregs were subsequently used for RNA sequencing analysis. Ingenuity Pathway Analysis showed upregulation of proinflammatory pathways (Th-1/Th-2) in iTregs following CPI-1205 treatment (Supplemental Figure 4A). Examination of the differentially expressed genes demonstrated an increase in effector cytokines and chemokines, whereas Tregspecific factors such as FoxP3, Bach2, and neuropilin 1 (Nrp1) were decreased following CPI-1205 treatment (Supplemental Figure 4B). We subsequently examined the cytokine levels in the culture supernatant of the iTregs that were differentiated with and without CPI-1205. Proinflammatory cytokines identified in the RNA sequencing analysis were significantly increased in the supernatant, confirming the gene signature of RNA sequencing data (Supplemental Figure 4C). Collectively, these data showed that CPI-1205 treatment influences the differentiation of iTregs and attenuates their suppressive activity. CPI-1205 can also skew the phenotype of iTregs to proinflammatory cytokine, producing effector-like T cells. In our study, we noted that EZH2 inhibition leads to decreased expression of not only FoxP3 but also other critical genes such as $N r p 1$ and Bach2, which are required for Treg stability (16-18). CPI-1205-treated Tregs seem to acquire the phenotype of so-called exFoxP3 cells, which were previously shown to have an activated memory phenotype (19). Further, our findings tie well with a recent report that showed that IFN- $\gamma$ production by a subset of Nrp1-deficient Tregs triggers instability of neighboring Tregs and restores antitumor immunity (16).

EZH2 inhibition has been shown to enhance Teff differentiation by releasing repressive marks on key cytokine and transcription factors; however, whether EZH2 inhibition affects the cytotoxicity of the $\mathrm{T}$ cells remains unknown. We investigated the effect 
A

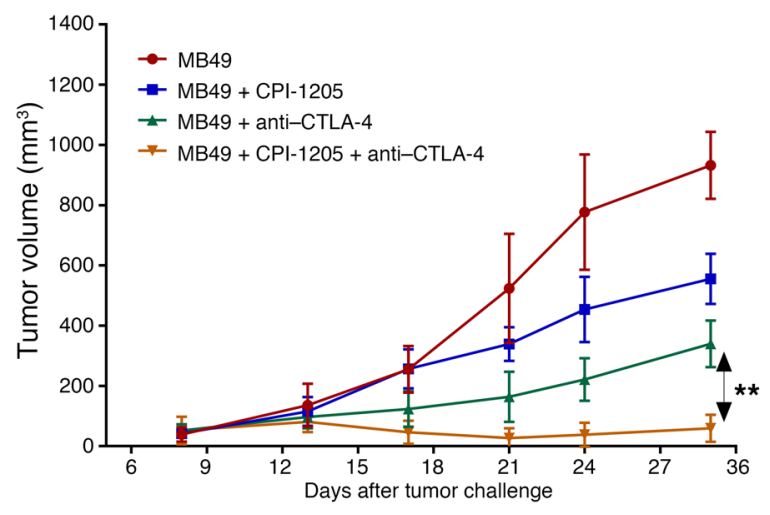

C

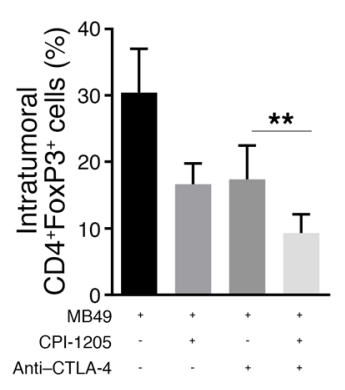

E

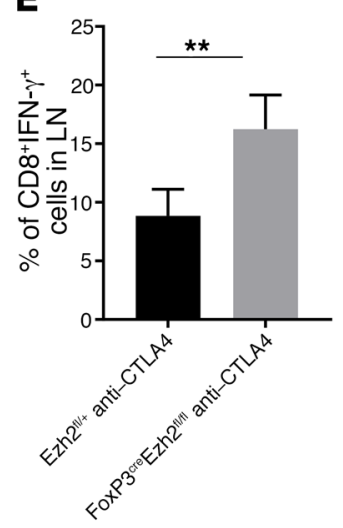

$\mathbf{F}$

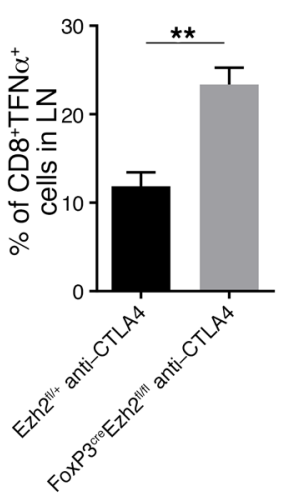

B

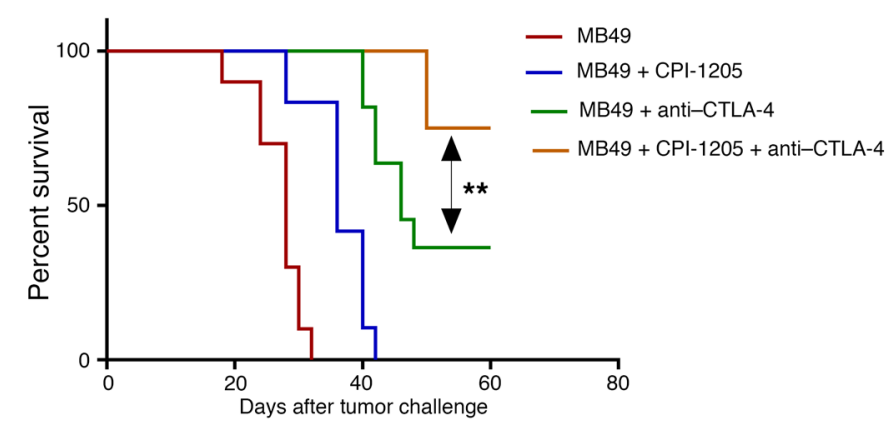

D

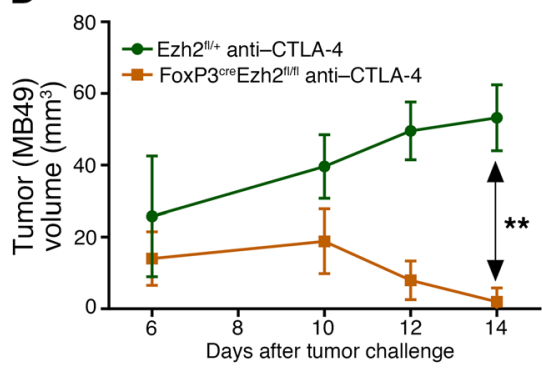

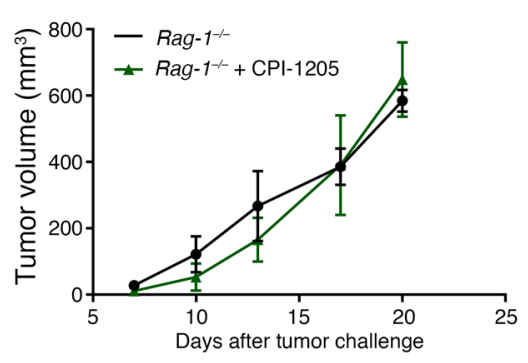

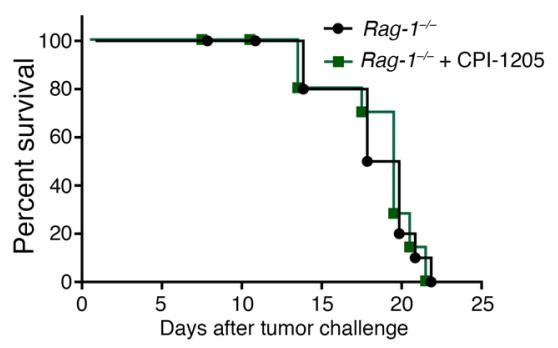

Figure 3. Blocking expression of EZH2 mediated by anti-CTLA-4 using CPI-1205 increases the effectiveness of anti-CTLA-4 therapy. Tumor growth (A), survival (B), and percentages of intra-tumoral CD4+CD25+FoxP3 ${ }^{+}$Tregs, $C D 4^{+} I \mathrm{COS}^{+} \mathrm{T}^{+}$-bet ${ }^{+}$, and $\mathrm{CD} 8^{+} \mathrm{IFN}-\gamma^{+}$cells (C) in MB49 tumor-bearing mice treated with vehicle, anti-CTLA-4, CPI-1205, or the combination. Tumor growth (D) and percentages of CD4+IFN- $\gamma^{+}$cells and CD8 $8^{+}$TNF- $\alpha^{+}$cells in the lymph nodes (LN) (E) of MB49 tumor-bearing EZH2 fl/ $^{f /}$ and FoxP3 ${ }^{\text {Cre } E Z H 2^{f l / f l}}$ mice treated with anti-CTLA-4 on days 7 and 9. (F) Tumor growth and survival of MB49 tumor-bearing $\operatorname{Rag}^{-1 /-}$ mice treated with vehicle or CPI-1205. Data are representative of 3 independent experiments; $n=10$ in each group; 1-way ANOVA was used to determine significance between the groups; ${ }^{* *} P<0.01$.

of CPI-1205 on effector T cell function. We performed a cytotoxicity assay using human peripheral $\mathrm{CD} 4^{+} \mathrm{T}$ cells and $\mathrm{CD} 8^{+} \mathrm{T}$ cells. Purified naive $\mathrm{CD}^{+}$and total $\mathrm{CD} 8^{+} \mathrm{T}$ cells were preactivated with plate-bound anti-CD3 and soluble anti-CD28 for 6 days in the presence or absence of CPI-1205. Activated T cells were then washed and cocultured with Nalm-6 target tumor cells and blinatumomab overnight as previously described (20) in the presence or absence of CPI-1205. Target cell death was determined by summing the percentage of apoptotic (Annexin $\mathrm{V}^{+}$) and dead or dying $\left(7 \mathrm{AAD}^{+}\right)$target (CellTrace Violet $\left.{ }^{+}\right)$cells after overnight culture. We found that CPI-1205 treatment of human $\mathrm{CD}^{+}$and
$\mathrm{CD} 8^{+} \mathrm{T}$ cells enhanced the cytotoxicity of effector $\mathrm{T}$ cells, leading to efficient target cell lysis at low effector-to-target ratios (Figure $1 \mathrm{E}$ and Supplemental Figure 5, A and B). There is a possibility that increased target cell lysis is secondary to CPI-1205-mediated phenotypic changes in target cells. However, in the cytotoxicity assay, the Nalm- 6 target tumor cells were exposed to CPI- 1205 for less than 20 hours. Such short exposure of tumor cells to CPI-1205 is not expected to have a transcriptional impact that could lead to phenotypic changes. Furthermore, increased IFN- $\gamma$ and TNF- $\alpha$ levels were noted in the supernatant of activated human $\mathrm{T}$ cells following CPI-1205 treatment, which suggested that these cells 
have enhanced effector functions (Supplemental Figure 6, A and B). Collectively, we found that genetic depletion and pharmacologic inhibition of EZH2 alters the functional phenotype of Tregs and enhances the cytotoxicity function of Teffs, which enables an improved antitumor response.

T cell activation by CD28 signaling induces EZH2 expression (1). We postulated that exaggerated CD28 signaling due to antiCTLA-4 therapy would increase EZH2 expression in T cells. To test this hypothesis, we isolated peripheral $\mathrm{CD} 4^{+} \mathrm{T}$ cells from patients with metastatic melanoma who received ipilimumab as the first line of therapy for metastatic disease, at baseline and after 3 doses of ipilimumab therapy. RNA sequencing analysis showed differential expression of histone-modifying enzymes (Figure 2A). Matched, paired analysis of histone-modifying enzymes revealed an increase in EZH2 expression in peripheral $\mathrm{CD}^{+} \mathrm{T}$ cells following ipilimumab therapy compared with baseline levels (Figure 2B). Next, we confirmed EZH2 expression in peripheral $\mathrm{CD} 4^{+} \mathrm{T}$ cells isolated from patients with metastatic prostate cancer at baseline and after 3 doses of ipilimumab (21). Like in patients with metastatic melanoma, we found increased EZH2 expression in CD $4^{+} \mathrm{T}$ cells following ipilimumab therapy (Figure 2C), which inversely correlated with prostate-specific antigen progression (Supplemental Figure 7, A and B). Next, we evaluated EZH2 expression on human $\mathrm{CD} 4^{+}$effector T cells $\left(\mathrm{CD} 4^{+} \mathrm{CD} 45 \mathrm{RO}^{-} \mathrm{CD} 45 \mathrm{RA}^{+} \mathrm{CCR} 7^{-}\right)$, Tregs $\left(\mathrm{CD} 4^{+} \mathrm{CD} 25^{+} \mathrm{FoxP}^{+}\right)$, and $\mathrm{CD}^{+} \mathrm{T}$ cells sorted from peripheral blood at baseline and after ipilimumab therapy. We noted that EZH2 expression increases in all $3 \mathrm{~T}$ cell subtypes following ipilimumab treatment (Supplemental Figure 8). To further elucidate the direct role of CTLA-4 signaling in EZH2 expression in T cells, we investigated EZH2 levels in T cells derived from CTLA$4^{-/-}$mice compared with WT littermate controls. We noted increased EZH2 levels in both $\mathrm{CD}^{+}$and $\mathrm{CD}^{+} \mathrm{T}$ cells in CTLA$4^{-/-}$mice compared with controls (Supplemental Figure 9). Altogether, we noted that blockade of CTLA-4 signaling enhances EZH2 expression in human and murine $\mathrm{T}$ cells. Because of the known effect of CTLA- 4 on T cell priming and CD28 signaling, we focused on the role of EZH2-mediated $\mathrm{T}$ cell function in anti-CTLA-4 therapy.

We evaluated whether the combination of anti-CTLA-4 with CPI-1205 increases the effectiveness of anti-CTLA-4. We treated MB49 (bladder) and B16-F10 (melanoma) tumorbearing C57BL/6 mice with anti-CTLA-4 and the EZH2 inhibitor CPI-1205 as monotherapies and in combination. We found that the combination therapy significantly reduced tumor growth and increased survival in both the MB49 and B16-F10 models compared with anti-CTLA-4 monotherapy (Figure 3, A and B and Supplemental Figure 10A). On interrogating the tumor immune environment, we noted that combination therapy with CPI-1205 plus anti-CTLA-4, as compared with monotherapy with CPI- 1205 or anti-CTLA-4, strikingly reduced the percentage of $\mathrm{CD}^{+}{ }^{+} \mathrm{FoxP} 3^{+}$Tregs and increased the percentage of intratumoral effector CD $4^{+} \mathrm{ICOS}^{+} \mathrm{T}-$ bet $^{+}$and CD $8{ }^{+} \mathrm{IFN}-\gamma^{+}$ cells in both the murine models (Figure $3 \mathrm{C}$ and Supplemental Figure 10B), which resulted in an increased ratio of Teffs to Tregs (Supplemental Figure 10C). Furthermore, MB49 tumor-bearing FoxP3 ${ }^{\text {cre }} E Z H 2^{f / f l}$ mice that received anti-CTLA-4 had com- plete tumor rejection (Figure 3D) and increased percentages of $\mathrm{CD}^{+} \mathrm{IFN}-\gamma^{+}$and $\mathrm{CD} 8^{+} \mathrm{TNF}-\alpha^{+}$cells (Figure $3 \mathrm{E}$ ) compared with WT controls. We used Rag1 ${ }^{-/-}$C57BL/6 mice, which have no mature lymphocyte population, to determine whether the antitumoral effect of combination therapy is exclusively due to effector immune response or is an additive effect of CPI-1205

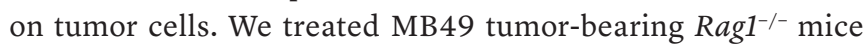
with CPI-1205. We did not notice any significant difference in tumor growth and survival in CPI-1205-treated $\mathrm{Rag1}^{-/-}$mice compared with the untreated group (Figure $3 \mathrm{~F}$ ), suggesting that EZH2 has no effect on tumor growth in the MB49 bladder cancer model. As EZH2 regulates expression of Th-1 chemokines such as CXCL9 and CXCL10 in the tumor cells (14), we performed in vitro (cell line) and in vivo (murine tumor model) experiments to assess the contribution of CXCL9 and CXCL10 in our system. We observed an increase in Cxcl9 (in B16-F10 cells) and Cxcl10 (in MB49 cells) following stimulation with IFN- $\gamma$ plus CPI-1205 in vitro (Supplemental Figure 11, A and B). Additionally, whereas CPI-1205 treatment increased the expression of $\mathrm{Cxcl} 9$ and $\mathrm{Cxcl1O}$ expression in MB49 tumor-bearing mice, combination treatment with the anti-CTLA-4 antibody plus CPI-1205 did not further increase tumor Cxcl9 or Cxcl1O as compared with the single-agent CPI-1205 treatment group (Supplemental Figure 11, C and D). Overall, these observations suggest that although CPI-1205 treatment might enhance chemokine-mediated $\mathrm{T}$ cell infiltration of tumors, the latter mechanism does not seem to substantially contribute to the enhanced antitumor immunity observed with the combination therapy. We also evaluated the contribution of other immune cell subsets such as B cells and myeloid cells in CPI-1205-mediated antitumor immunity. Although we did not note any difference in B cell abundance, CPI-1205 treatment had an influence on certain subsets of myeloid cells, which will warrant further investigations.

In conclusion, we demonstrated the direct role of EZH2mediated reprogramming of $\mathrm{T}$ cells in antitumor immunity, especially in the context of anti-CTLA-4 therapy. Ipilimumab leads to a compensatory increase in EZH2 expression in T cells, whereas inhibition of EZH2 improves response to anti-CTLA-4 through modulation of tumor cytotoxic effector $\mathrm{T}$ cells and altering the phenotype of Tregs into effector-like T cells. We did not observe any immune-related toxicities in mice treated with anti-CTLA-4 plus CPI-1205. Ipilimumab has been safely given to patients in combination with other immune checkpoint therapy such as nivolumab with a manageable side effect profile. Importantly, CPI-1205 is well-tolerated when given by continuous daily dosing in patients with no dose-limiting toxicities. Thus, the mechanistic insight gathered in this study provides a strong rationale to initiate a clinical trial with CPI-1205 plus ipilimumab in patients with primary or adaptive resistance to anti-CTLA-4 therapy.

\section{Methods}

Further information can be found in the Supplemental Methods and in Supplemental Figures 1-9.

Accession numbers. RNA sequencing data were deposited in the NCBI Gene Expression Omnibus database (GEO GSE114716, GSE114717). 
Statistics. All data are mean \pm SEM. A 2-tailed Student's $t$ test or 1-way ANOVA or Bonferroni multiple comparison tests were used to identify significant differences $(P<0.05)$ between treatment groups using GraphPad Prism 7 software. Ingenuity Pathway Analysis was done on data obtained from RNA sequencing using Ingenuity Pathway Analysis software (Qiagen). A $P$ value of less than 0.05 was considered statistically significant.

Study approval. Clinical protocol was approved by the IRB at The University of Texas MD Anderson Cancer Center (21). All animal experiments were conducted according to protocols approved by the Animal Resource Center at The University of Texas MD Anderson Cancer Center (IACUC 00000893-RN01).

\section{Author contributions}

PS was responsible for overall supervision and evaluation of all experimental studies. SG and PS designed the study. SG performed the experiments, analyzed the data, and wrote the manuscript. JZ, SA, XZ, LX, and JS provided technical assistance. AA and SKS eval- uated the clinical responses of metastatic prostate cancer patients. HZ performed the bioinformatics analysis. IA, JPA, PT, and PS provided critical input and reviewed the manuscript.

\section{Acknowledgments}

We thank the Immunotherapy Platform at MD Anderson Cancer Center for technical support. We also thank Spencer C. Wei and Ashwin Jaiswal for helpful discussions. We thank Yuji Miura and Bill Bradley for technical assistance. JPA and PS are codirectors of the Parker Institute for Cancer Immunotherapy. PS received funding through the National Institutes of Health (grant R01CA163793) and the Parker Institute for Cancer immunotherapy.

Address correspondence to: Padmanee Sharma, The University of Texas MD Anderson Cancer Center, 1515 Holcombe Boulevard, Houston, Texas 77030, USA. Phone: 713.792.3250; Email: padsharma@mdanderson.org.
1. DuPage M, et al. The chromatin-modifying enzyme Ezh2 is critical for the maintenance of regulatory $\mathrm{T}$ cell identity after activation. Immunity. 2015;42(2):227-238.

2. Cao R, et al. Role of histone H3 lysine 27 methylation in Polycomb-group silencing. Science. 2002;298(5595):1039-1043.

3. Margueron R, Reinberg D. The Polycomb complex PRC2 and its mark in life. Nature. 2011;469(7330):343-349.

4. Arvey A, van der Veeken J, Samstein RM, Feng Y, Stamatoyannopoulos JA, Rudensky AY. Inflammation-induced repression of chromatin bound by the transcription factor Foxp3 in regulatory T cells. Nat Immunol. 2014;15(6):580-587.

5. Yang XP, et al. EZH2 is crucial for both differentiation of regulatory $\mathrm{T}$ cells and $\mathrm{T}$ effector cell expansion. Sci Rep. 2015;5:10643.

6. Kwon HK, Chen HM, Mathis D, Benoist C. Different molecular complexes that mediate transcriptional induction and repression by FoxP3. Nat Immunol. 2017;18(11):1238-1248.

7. Tumes DJ, et al. The polycomb protein Ezh2 regulates differentiation and plasticity of CD4(+) T helper type 1 and type 2 cells. Immunity. 2013;39(5):819-832.

8. Wei G, et al. Global mapping of H3K $4 \mathrm{me} 3$ and
$\mathrm{H} 3 \mathrm{~K} 27 \mathrm{me} 3$ reveals specificity and plasticity in lineage fate determination of differentiating CD4+ T cells. Immunity. 2009;30(1):155-167.

9. Zhang Y, et al. The polycomb repressive complex 2 governs life and death of peripheral $\mathrm{T}$ cells. Blood. 2014;124(5):737-749.

10. Kanno Y, Vahedi G, Hirahara K, Singleton K, O'Shea JJ. Transcriptional and epigenetic control of T helper cell specification: molecular mechanisms underlying commitment and plasticity. Annu Rev Immunol. 2012;30:707-731.

11. Coit $\mathrm{P}$, et al. Epigenetic reprogramming in naive CD4+ T cells favoring $\mathrm{T}$ cell activation and non-Th1 effector $\mathrm{T}$ cell immune response as an early event in lupus flares. Arthritis Rheumatol. 2016;68(9):2200-2209.

12. Sarmento OF, et al. The role of the histone methyltransferase enhancer of zeste homolog 2 (EZH2) in the pathobiological mechanisms underlying inflammatory bowel disease (IBD). J Biol Chem. 2017;292(2):706-722.

13. Nagarsheth $\mathrm{N}$, et al. PRC2 epigenetically silences Th1-type chemokines to suppress effector T-cell trafficking in colon cancer. Cancer Res. 2016;76(2):275-282.

14. Peng D, et al. Epigenetic silencing of TH1-type chemokines shapes tumour immunity and immu- notherapy. Nature. 2015;527(7577):249-253.

15. Zingg D, et al. The histone methyltransferase Ezh2 controls mechanisms of adaptive resistance to tumor immunotherapy. Cell Rep. 2017;20(4):854-867.

16. Overacre-Delgoffe AE, et al. Interferon- $\gamma$ drives Treg fragility to promote anti-tumor immunity. Cell. 2017;169(6):1130-1141.e11.

17. Roychoudhuri R, et al. BACH2 represses effector programs to stabilize $\mathrm{T}$ (reg)-mediated immune homeostasis. Nature. 2013;498(7455):506-510.

18. Delgoffe GM, et al. Stability and function of regulatory $\mathrm{T}$ cells is maintained by a neuropilin-1-semaphorin-4a axis. Nature. 2013;501(7466):252-256.

19. Zhou X, et al. Instability of the transcription factor Foxp3 leads to the generation of pathogenic memory T cells in vivo. Nat Immunol. 2009;10(9):1000-1007.

20. Mølhøj M, et al. CD19-/CD3-bispecific antibody of the BiTE class is far superior to tandem diabody with respect to redirected tumor cell lysis. Mol Immunol. 2007;44(8):1935-1943.

21. Subudhi SK, et al. Clonal expansion of CD8 T cells in the systemic circulation precedes development of ipilimumab-induced toxicities. Proc Natl Acad Sci U S A. 2016;113(42):11919-11924. 